\title{
Ultra-Cool Very Low-Mass Binaries
}

\author{
Eduardo L. Martín \\ Division of Geology and Planetary Sciences, Caltech, MC 150-21, \\ Pasadena, CA 91125 \\ Gibor Basri \\ Astronomy Department, MC 3411, Univ. of California, Berkeley, CA \\ 94720
}

\begin{abstract}
Observations of ultra-cool (spectral type later than M6) binaries are summarized. Only a few systems are known, and all have been discovered in the past few years. We describe each of these discoveries. Despite their small numbers, some interesting trends among the binaries may be emerging. Ultra-cool binaries have a frequency similar to but perhaps a little less than stars (binary fraction $\sim 20 \%$ ) and have mass ratios closer to unity, with respect to solar-type stars. Neither of these results can be considered firm, as there are far too few systems known, and observational biases would tend to move trends in these directions. There also seems to be a trend towards smaller separations in lower mass systems, which is less subject to observational biases. We discuss how these results fit into current ideas about binary formation, and favor a fragmentation scenario.
\end{abstract}

\section{Introduction}

Ultra-cool dwarfs will be defined as high-gravity objects (log g $>3.5$ ) with spectral type later than M6 (effective temperature cooler than about $2800 \mathrm{~K}$ ). They can have masses above or below the substellar limit. This paper is mostly about multiple systems in which the primary qualifies as an ultra-cool dwarf. At the age of the Pleiades cluster $(\sim 120 \mathrm{Myr})$, the substellar limit is located at spectral type M6.5 (Martín et al. 1996). For younger ages, it can be as warm as M6, and for older ages it becomes progressively cooler, dropping below M9.5 at about one Gyr according to evolutionary models (Chabrier et al. 2000). Generally, substellar objects are warmer when they are younger because they radiate mainly gravitational energy. Flux limited surveys like DENIS and 2MASS are biased toward finding young brown dwarfs and nearly-equal mass binaries. These surveys are finding so many ultra-cool dwarfs that systematic studies of their binary frequency are being initiated. Binarity studies have also started in the Pleiades cluster where about 30 brown dwarfs have been identified, and among low mass Hyades stars.

The formation of binary and higher-order multiple systems has been recognized as a common product of star formation. It is widely accepted that the 
formation of a single star with a planetary system like our own is more the exception than the rule. The majority (57\%) of G- and K-type dwarfs in the solar vicinity have H-burning companions (Abt \& Levy 1976; Duquennoy \& Mayor 1991). M-dwarf primaries were thought to have a lower occurrence of companions (38\%; Henry \& McCarthy 1990; Fisher \& Marcy 1992; Reid \& Gizis 1997), although recent work may be raising that (see Mayor, this volume). Those results raise the following questions: What is the frequency of companions for very low-mass (VLM) primaries? Is there a trend of lower binary frequency for lower primary mass?

One motivation to search for binary brown dwarfs is to learn about the origin of these objects. Free-floating brown dwarfs can form in several ways. They could be ejected from an unstable multiple system. This would lead to a very low brown dwarf binary fraction, as such a process would typically eject single objects, and disrupt most brown dwarf binaries (except the very hard ones). Condensation within a circumstellar disk, which would be a higher mass analogue to the formation of a planet, should also probably yield a low binary fraction among brown dwarfs, because planets are not generally expected to be double (in our solar system there is only the dubious example of the PlutoCharon system). Fragmentation and collapse of a small and molecular core would be the lower mass analogue to the formation of a star, would probably yield a significant binary fraction among brown dwarfs, in analogy to stars.

Another motivation for searching for brown dwarf binaries is that they can provide dynamical masses, which are powerful tests of evolutionary models of substellar objects, and can help to derive an observational mass-luminosity relationship below the substellar limit. Last but not least, any faint physical companion to a brown dwarf must be also be nonstellar: another brown dwarf, a planet or a faint compact object (white dwarf, neutron star, black hole). Brown dwarfs offer the opportunity of finding very cool companion objects outside the solar-system, which can be used as benchmarks for further studies of extrasolar planets.

\section{Searches for Ultra-Cool Binaries}

For more than a decade, a number of groups have conducted searches for companions to cool and ultra-cool dwarfs using near-infrared cameras. Many of the results of such surveys have remained unpublished due to the unfortunate tendency of researchers to publish only positive results. Before discussing the ultra-cool systems, it is worthwhile to briefly survey the results of the searches for ultra-cool companions to warmer stars. This was one of the favored methods of searching for brown dwarfs before 1995. For early to mid $\mathrm{M}$ dwarf primaries, the incidence of VLM companions is relatively low. Three visible brown dwarf companions are known with early M companions: Gl 229B (the famous first methane dwarf; Nakajima et al. 1995); G196-3B, a lithium bearing L brown dwarf (Rebolo et al. 1998); and Gl 570D, another methane dwarf in a quadruple system with a $\mathrm{K}$ primary and $\mathrm{M}$ close binary (Burgasser et al. 2000). The projected separations in these 3 systems are (respectively) $40 \mathrm{AU}, 300 \mathrm{AU}$, and $1500 \mathrm{AU}$. 
A similar number of radial velocity substellar companions are known with more massive primaries (and even lower occurrence statistics); they are necessarily only a few AU from their primaries (because of the detection method). These include HD 114762 (Latham et al. 1989), and HD 29587 and 127506 (Halbwachs et al. 2000). Finally, there recently have been found a few VLM wide companions to more massive stars. Two of them, h CrB C and Gl 417B (Kirkpatrick et al. 1999) have $\mathrm{G}$ primaries, with projected separations of 3600 and $2000 \mathrm{AU}$. Two possible brown dwarf companions to A stars are known: GD 165B (Becklin and Zuckerman 1988; the A star is now a white dwarf) and HR 7329 (Lowrance et al. 2000). These have projected separations of 300 and 200 AU respectively. Each of these was found in surveys containing large numbers of targets with non-detections. It is clear that the binary incidence for ultra-cool companions in high mass ratio systems is much smaller than for stellar binaries; probably $1 \%$ or less for mass ratios greater than about 10.

More than two hundred ultra-cool dwarfs are now known. Typically they are detected with low-spatial resolution imaging (FWHM from one to a few arcsec). In order to search for binaries deep high resolution images are necessary. Radial velocity and astrometry are other powerful techniques that can be used to detect binaries, but they have only recently been exploited for ultra-cool dwarf primaries due to the faintness of these objects. The number of ultra-cool dwarfs has increased so much in the last few years that they constitute a sample from which preliminary binary statistics can be derived.

A numerous population of free-floating brown dwarfs has been discovered in the Pleiades cluster (e.g. Zapatero Osorio et al. 1999; Pinfield et al. 2000). G and K-type Pleiades members have a distribution of both orbital periods and massratios similar to those of field G-dwarf binaries (Bouvier et al. 1997). Thus, it seems that the binary properties of the Pleiades stars might not be very different from those of the disk population. Martín et al. (2000) carried out a search for companions among 34 very low-mass Pleiades members $\left(\mathrm{M} \leq 0.09 \quad \mathrm{M}_{\odot}\right)$ using high-spatial resolution images obtained with the Hubble Space Telescope and the adaptive optics system of the Canada-France-Hawaii telescope. Only one binary was resolved but follow-up observations did not confirm its membership to the Pleiades cluster.

The first two free-floating brown dwarfs in the solar neighborhood confirmed with the lithium test (Magazzù et al. 1993) were subject to a mini-survey with HST/NICMOS just before it run out of coolant. No companion was found around Kelu 1 (discovered by Ruiz et al. 1997 in a proper motion study) down to a limiting mass of about 7 Jupiters. On the other hand, DENIS-P J1228-15 (discovered by Delfosse et al. 1997 in a pilot study of DENIS data) was found to be a binary (see next section). Koerner et al. (1999) presented images obtained with the Keck $10 \mathrm{~m}$ telescope where they found an elongation in the point spread function (PSF) of DENIS-P J1228-15 but not in that of Kelu 1. They found that two more L dwarfs had elongated PSFs. Martín et al. (2000a) have started a search for ultra-cool binaries using adaptive optics on large telescopes. The first binary discovered is Gl 569b. Gl 229B and Gl $105 \mathrm{C}$ are not binaries ( $F W H M=0.05$ arcsec in the $\mathrm{H}$-band, $\Delta \mathrm{H}=5 \mathrm{mag}$ ). Several programs have been approved with HST to search for binaries among ultra-cool dwarfs in the field 
and in open clusters. We expect a flurry of discoveries in the next few years, and a big improvement in the statistics of multiplicity properties.

\section{Discoveries of Ultra-Cool Binaries}

The known ultra-cool binaries are so few that they can be presented one by one in some detail. This list will undoubtedly be obsolete by the time it appears, as new discoveries are coming quickly. We will use a chronological order:

- LHS 1070: Leinert et al. (1994) reported the discovery of a companion to the nearby star LHS $1070(\mathrm{~d}=7.5 \mathrm{pc})$. The angular separation was 1.1 arcsec, corresponding to $8.2 \mathrm{AU}$. The companion is itself a nearly-equal mass closer binary, with separation $0.3 \operatorname{arcsec}(2.2 \mathrm{AU})$. The spectral type of LHS $1070 \mathrm{~A}$ is M5.5, and that of LHS $1070 \mathrm{~B}$ and C are M8 (Leinert et al. 2000). The orbital period could be about 12 years.

- PPl 15: The faintest object discovered in the survey of Stauffer, Hamilton and Probst (1994) in the Pleiades, has a spectral type of M6.5 (Martín, Rebolo \& Zapatero Osorio 1996), and was found to have lithium by Basri, Marcy \& Graham (1996). However, they did not realize that it is a binary until this possibility was pointed out by Zapatero Osorio, Martín \& Rebolo (1997) on the basis of its location in the cluster sequence. Basri \& Martín (1998) reanalized the data of Basri et al. (1996) and reported the detection of double-lined splitting in the cross-correlation functions of red orders in the Keck/HIRES spectra. Basri \& Martín (1999) demostrated that PPl 15 is a spectroscopic binary with a high mass ratio (q 0.9), a short period of 5.8 days and a large eccentricity $(\mathrm{e}=0.4 \pm 0.05)$. In the Pleiades, stellar binaries with orbital periods shorter than 7 days have circular orbits (Mermilliod et al. 1992). The eccentric orbit of PPl 15 breaks the rules of stellar binaries and indicates that hard binaries are born with high eccentricity and the orbits circularize in a short timescale as predicted by tidal force calculations (Zahn \& Bouchet 1989). The tidal torque $T$ is proportional to $\mathrm{M}^{2}$ and $\mathrm{R}^{2}$. Hence, it is much weaker in VLM objects with small radii, such as brown dwarfs.

- CFHT-P1 18: This Pleiades brown dwarf candidate found by Bouvier et al. (1998) was resolved as a binary with 0.33 arcsecond separation with HST (Martín et al. 1998). The spectral type of the primary is about M8. Martín et al. (2000b) applied the lithium test with negative result. Weak $\mathrm{H}_{\alpha}$ emission in comparison with Pleiades members of the same spectral type suggests that the age of CFHT-Pl 18 could be rather old. It is likely a binary with VLM components (probably stellar) located on the line of sight to the Pleiades. It is not possible to estimate an orbital period because the distance to this object is unknown.

- DENIS-P J1228-15: The first DENIS brown dwarf with lithium (Delfosse et al. 1997; Martín et al. 1997; Tinney et al. 1997). It has a spectral type of L4.5 (Martín et al. 1999b). NIC1/HST imaging resolved it into two component of nearly equal brightness $\left(\Delta \mathrm{m}_{1.65 \mu m}=0.15 \mathrm{mag}\right)$. The angular 
separation is 0.275 arcsec and $\mathrm{PA}=41^{\circ}$. The semimajor axis is about $5 \mathrm{AU}$ (for a distance of $18 \mathrm{pc}$, Dahn et al. 2000) and the orbital period about 35 years. Koerner et al. (1999) and one of us (EM) confirmed that the system is physical because the separation remained almost the same in Keck/NIRC images obtained in February 1999 and February 2000. The proper motion of this brown dwarf is $0.21 \operatorname{arcsec} \mathrm{yr}^{-1}$.

- DENIS-P J0205-11: The coolest of the first three DENIS L dwarfs (Delfosse et al. 1997), it has a spectral type of L5 (Martín et al. 1999), but does not show lithium (Martín et al. 1997) implying that it is more massive and older than DENIS-P J1228-15. Nevertheless, it is likely a brown dwarf because it has a temperature of $\sim 1700 \mathrm{~K}$ (Basri et al. 2000), which is cooler than the bottom of the main sequence (Burrows et al. 1997; Chabrier et al. 2000). Koerner et al. (1999) imaged it in the K-band at 2 different epochs and found elongated PSFs with a separation of 0.5 arcsec. This result requires confirmation with more epochs or with higher spatial resolution images. If real, the binary would be nearly-equal mass and have a projected separation of $9.2 \mathrm{AU}$ and a period of about 80 years.

- 2MASSW J1146+22: A lithium L dwarf like DENIS-P J1228-15, discovered in the 2MASS survey (Kirkpatrick et al. 1999). The spectral type is L3, so it is somewhat warmer than the other two binaries discussed before. Koerner et al. (1999) imaged it in the K-band at 3 different epochs and found elongated PSFs with a separation of 0.3 arcsec. The binary has a nearly-equal mass, a projected separation of $7.6 \mathrm{AU}$ and a period of about 65 years.

- GJ 569: A nearby (d=9.8 pc) M3 star with high levels of chromospheric and coronal activity. Forrest, Skrutskie \& Shure (1988) reported a possible brown dwarf companion separated 5" from the primary. Henry \& Kirkpatrick (1990) obtained a low resolution spectrum and classified it as an M8.5 dwarf. Magazzù et al. (1993) did not detect lithium in Gl 569 B, even though its position in the HR diagram suggest that it is a very young object. Martín et al. (2000b) found that Gl 569 B is a binary with a separation of $0.1 \operatorname{arcsec}(1 \mathrm{AU})$. Follow-up imaging obtained with the Keck/AO facility are shown in Figure 1. Orbital motion is clearly detected, indicating that the orbital period is about 2 years. This system could yield the first dynamical masses for ultra-cool dwarfs.

\section{Properties of Ultra-Cool Binaries}

In our Pleiades binary survey (Martín et al. 2000a), no resolved binary bonafide members of the cluster with separations larger than $0.2 \operatorname{arcsec}$ (a 27 AU) were found. Nevertheless, we found evidence for a binary sequence in the colormagnitude diagrams, in agreement with the results of Steele \& Jameson (1995) for higher mass stars. We applied the lithium test to two objects: CFHT-Pl-16, which lies in the cluster binary sequence but is unresolved in images obtained with the Hubble Space Telescope; and CFHT-Pl-18, which is binary with 0".33 


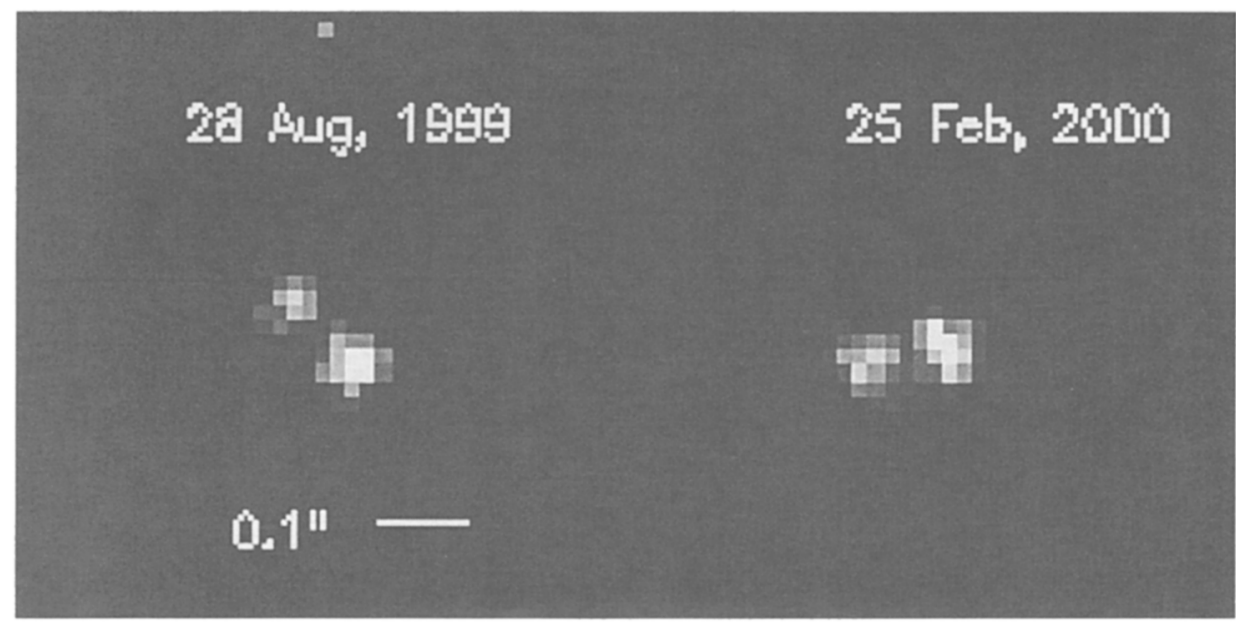

Figure 1. Adaptive Optics Images of Gl 569B obtained with the Natural Guide Star system at Keck. In the figure North is up, East is to the left.

separation (Martín et al. 1998). The first object passed the test, but the second object did not. We concluded that CFHT-Pl-16 is likely a Pleiades brown dwarf binary with separation $<11 \mathrm{AU}$, and that CFHT-Pl-18 is a foreground system. A comparison of the multiplicity statistics of the Pleiades very low-mass stars and brown dwarfs with that of $\mathrm{G}$ and $\mathrm{K}$-type main sequence stars in the solar neighborhood (Duquennoy \& Mayor 1991), indicates that 3.4 binaries were expected with separation $>27$ AU, but none was found. Martín et al. (2000a) concluded that there is some evidence for a deficiency of wide binary systems among the Pleiades very low-mass members.

On the other hand, the discovery that PPl 15 is a double-lined brown dwarf binary with a period of only 5.8 days (Basri \& Martín 1999) is surprising because the chance of finding such a hard binary in a Duquennoy \& Mayor (1991) population is only $10 \%$ (Heacox 1998). Among the first brown dwarfs discovered in the field, there are already 3 brown dwarf binaries with separations in the range 5-10 AU as discussed in the previous section. It is noteworthy that all these 3 binaries were found near the resolution limit of the images. Wider binaries could have been easily detected, but closer binaries could not. The Pleiades and field results are consistent and suggest that ultra-cool binaries are less common ( $\sim 20 \%$ binary frequency) and prefer smaller separations than stellar binaries. There is also a tendency to find nearly-equal mass binaries. This could be partially due to a bias of the observations because such binaries are more luminous and are easier to detect.

We and others have started spectroscopic and near-infrared imaging surveys aimed at detecting ultra-cool binaries. Particularly interesting targets are the methane dwarfs because fainter companions would be cooler, and would approach the temperatures of extra-solar planets. In a sample of 14 methane dwarfs, we have found only one companion, which is 3 magnitudes fainter than the primary (SDSS 134664-00; Tsevetanov et al. 2000) in the K-band, and has 
an angular separation of 1.4 arcsec. However, a VLT/ISAAC spectrum obtained by J.L. Beuzit upon request from one of us (EM) does not show methane in the faint object. The spectrum is red and featureless. It is likely an extragalactic object. Typical distances to these methane dwarfs are 10 to $15 \mathrm{pc}$. Thus, we find no companions with separations larger than $\sim 30 \mathrm{AU}$, in agreement with our results for the Pleiades cluster.

\section{Origins of Ultra-Cool Binaries}

- Capture: This scenario is not supported by observations because brown dwarfs are rare $(<1 \%)$ as companions to stars within $5 \mathrm{AU}$ (brown dwarf desert; Marcy \& Butler 1998; Halbwachs et al. 2000), but common ( 20\%) as companions to ultracool dwarfs. It also fails to explain why wide brown dwarf binaries are rare. On the other hand, capture could be a viable mechanism to explain wide brown dwarf companions to stars like Gl 570D (Burgasser et al. 2000). As noted below, the distinction between capture and fragmentation becomes a bit fuzzy when one considers formation in a small $\mathrm{N}$ cluster.

- Disk Fragmentation: It tends to produce high mass ratio systems. Not supported: brown dwarf companions to stars are rare as stated above.

- Fragmentation: This is the favoured mechanism for producing most binary stellar systems (Bate 2000) because it appears possible to form binaries with similar properties to those that are observed. Supported: the fraction of substellar systems which are binary may not be much lower than the fraction of stellar binaries. Numerical simulations of molecular cloud fragmentation have shown that slower rotating cloud cores form closer binaries than when the clouds are rapidly rotating (Boss 1993). Small cloud cores that start collapse from high initial densities form even closer binaries. This scenario may provide a natural way of explaining the absence of wide brown dwarf binaries and an excess of close systems.

A variant on this which is perhaps even more attractive is formation of single and binary VLM objects in small $\mathrm{N}$ clusters. There are several papers in this volume which discuss this process. The apparent tendency of such calculations to form a few very low mass objects early in the formation of interacting disks is positive, but the calculations have not been carried out far enough to assure that these objects survive. They would tend to be ejected later on if there are several more massive objects; only the tight couples among them would survive as binaries. Clarke (1996) has discussed binary formation in this scenario. One tends to require that $\mathrm{N}$ be only a few to be compatible with the stellar statistics. One prediction which seems to be upheld is that large mass ratio binaries should be rare. This theory also predicts that the binary fraction should decrease with primary mass; our preliminary results may support this (but it is premature to draw any firm conclusions).

In addition to formation scenarios, the brown dwarf binaries in the Pleiades and the field may have suffered dynamical evolution. Wide binaries can be disrupted 
due to dynamical interactions with stars. Such process has been advocated by Kroupa, Petr \& McCaughrean (1999) to explain the binary frequency of stars in the Trapezium cluster. An important test of this scenario is the binary frequency of hard binaries, which should be independent of primary mass. Another test is the binary frequency of brown dwarfs in very young associations and clusters. For example, Najita, Tiede \& Carr (2000) have identified a candidate wide brown dwarf binary in IC348. Spectroscopic follow-up is necessary to confirm it.

Acknowledgments. We thank the SOC for their invitation to give a review talk. We acknowledge helpful discussions with many colleagues: I. Baraffe, G. Chabrier, X. Delfosse, Th. Forveille, A. Ghez, P. Goldreich, B. Lane, G. Laughlin, D. Lin, M. Marley, among others.

\section{References}

Abt, H. A., \& Levitt, S. G. 1976, ApJS, 30, 273

Basiri, G., Marcy, G., \& Graham, J. 1996, ApJ, 458, 600

Basri, G., \& Martín, E. L. 1998, in ASP Conf. Series Vol. 134, Brown Dwarfs and Extrasolar Planets, ed. R. Rebolo, E. L. Martín \& M. R. Zapatero Osorio, 284

Basri, G., \& Martín, E. L. 1999, AJ, 118, 2460

Basri, G. et al. 2000, ApJ, in press

Bate, M. 2000, MNRAS, 314, 33

Becklin, E. E., \& Zuckerman, B. 1988, Nature, 336, 656

Boss, A. 1993, in "The Realm of Interacting Binary Stars", ed. J. Sahade, Kluwer Academic Publishers, 355

Bouvier, J., Rigaut, F., \& Nadeau, D. 1997, A\&A, 323, 139

Burrows, A. et al. 1997, ApJ, 491, 856

Burgasser, A. et al. 2000, ApJ, 531, L57

Chabrier, G., Baraffe, I., Allard, F., \& Hauschildt, P. 2000, ApJ, in press

Clarke, C. 1996, in ASP Conf. Series ,Vol. 90, The Origins, Evolution, and Destinies of Binary Stars in Clusters, ed. E. F. Milone \& J. C. Mermilliod, 242

Dahn, C. et al. 2000, in From Giant Planets to Cool Stars, ed. C. Griffith \& M. Marley, in press

Delfosse, X. et al. 1997, A\&A, 327, L25

Duquennoy, A., \& Mayor, M. 1991, A\&A, 248, 485

Fisher, D. A., \& Marcy, G. W. 1992, ApJ, 396, 178

Forrest, W. J., Skrutskie, M. F., \& Shure, M. 1988, ApJ, 330, L119

Halbwachs, J. L., Arenou, F., Mayor, M. Udry, S., \& Queloz, D. 2000, A\&A, 355,581

Henry, T. J., \& Kirkpatrick, D. J. 1990, ApJ, 354, L29

Henry, T. J., \& McCarthy, D. W. 1990, ApJ, 350, 334

Heacox, W. D. 1998, AJ, 115, 325

Kirkpatrick, J. D. et al. 1999, ApJ, 519, 802 
Koerner, D. W., Kirkpatrick, J. D., McElwain, M. W., \& Bonaventura, N. R. 1999, ApJ, 526, L25

Kroupa, P., Petr, M. G., \& McCaughrean, M. J. 1999, NewA, 4, 495

Latham, D.W., Mazeh, T., Stefanik, R.P., Mayor, M., Burki, G. 1989, Nature, 339,38

Leinert, Ch., Weitzel, N., Richichi, A., Eckart, A., \& Tacconi-Garman, L. E. 1994, A\&A, 291, L47

Leinert, Ch., Allard, F., Richichi, A., \& Hauschildt, P. 2000, A\&A, 353, 691

Lowrance, P.J., Schneider, G., Kirkpatrick, J.D. et al. 2000, astro-ph/0005047

Magazzù, A., Martín, E. L., \& Rebolo, R. 1993, ApJ, 404, L17

Marcy, G., \& Butler, R. P. 1998, ARA\&A, 36, 57

Martín, E. L., Rebolo, R., \& Zapatero Osorio, M. R. 1996, ApJ, 469, 706

Martín, E. L. et al. 1998, ApJ, 509, L113

Martín, E. L. et al. 1999a, 118, 2466

Martín, E. L., Brandner, W., \& Basri, G. 1999a, Science, 283, 1718

Martín, E. L., Koresko, Ch., Kulkarni, S., Lane, B., \& Wizinowich, P. 2000a, ApJ, 529, L37

Martín, E. L. et al. 2000a, ApJ, in press

Martín, E. L., Koresko, Ch., Kulkarni, S., Lane, B., \& Wizinowich, P. 2000b, ApJ, 529, L37

Mermilliod, J. C., Rosvick, J. M., Duquennoy, A., \& Mayor, M. 1992, A\&A, 265,513

Najita, J. R., Tiede, G. P., \& Carr, J. S. 2000, ApJ, in press

Nakajima, T., Oppenheimer, B. R., Kulkarni, S. R., Golimowski, D. A., Matthews, K., Durrance, S. T. 1995, Nature, 378, 463

Pinfield, D. J., Hodgkin, S. T., Jameson, R. F., Cossburn, M. R., Hambly, N. C., \& Devereux, N. 2000, MNRAS, 313, 347

Rebolo, R., Zapatero-Osorio, M. R., Madruga, S., Bejar, V. J. S., Arribas, S., Licandro, J. 1998, Science, 282, 1309

Reid, I. N., \& Gizis, J. E. 1997, AJ, 114, 1992

Ruiz, M. T., Leggett, S. K., \& Allard, F. 1997, ApJ, 491, L107

Stauffer, J. R., Hamilton, D., \& Probst, R. G. 1994, AJ, 108, 155

Steele, I. A., \& Jameson, R. F. 1995, MNRAS, 272, 630

Tinney, Ch., Delfosse, X., \& Forveille, Th. 1997, ApJ, 490, L95

Tsvetanov, Z. et al. 2000, ApJ, 531, L61

Zahn, J., \& Bouchet, L. 1989, A\&A, 223, 112

Zapatero Osorio, M. R., Martín, E. L., \& Rebolo, R. 1997, A\&A, 323, 105

Zapatero Osorio, M. R. et al. 1999, A\&AS, 134, 537 